\title{
A project of a two meter telescope in North Africa
}

\author{
Zouhair Benkhaldoun
}

Oukaimden Observatory, Cadi Ayyad University

\begin{abstract}
Site testing undertaken during the last 20 years by Moroccan researchers through international studies have shown that the Atlas mountains in Morocco has potentialities similar to those sites which host the largest telescopes in world. Given the quality of the sites and opportunities to conduct modern research, we believe that the installation of a $2 \mathrm{~m}$ diameter telescope will open new horizons for Astronomy in Morocco and north Africa allowing our region to enter definitively into the very exclusive club of countries possessing an instrument of that size. A state of the art astrophysical observatory on any good astronomical observation site should be equipped with a modern $2 \mathrm{~m}$-class, robotic telescope and some smaller telescopes. Our plan should be to operate one of the most efficient robotic $2 \mathrm{~m}$ class telescopes worldwide in order to offer optimal scientific opportunities for researchers and maintain highest standards for the education of students. Beside all categories of astronomical research fields, students will have the possibility to be educated intensively on the design, manufacturing and operating of modern state of the art computer controlled instruments. In the frame of such education and observation studies several $\mathrm{PhD}$ and dissertational work packages are possible. Many of the observations will be published in articles worldwide and a number of guest observers from other countries will have the possibility to take part in collaborations. This could be a starting point of an international reputation of our region in the field of modern astronomy.
\end{abstract}

